\title{
Motivos de abandono y no práctica de actividad físico- deportiva en adolescentes españoles: estudio Avena
}

\section{Motives of Doprout and Non Practice Physical Activity and Sport in Spanish Adolescents: The Avena Study}

\author{
Motivos de abandono e a não pratica físico-esportiva \\ em adolescentes espanhóis: estudo Avena
}

\author{
Alejandro César Martínez Baena*, Palma Chillón*, Miguel Martín-Matillas*, Isaac Pérez López*, Ruth Castillo**, Belén \\ Zapatera $^{* * *}$, Germán Vicente-Rodríguez ${ }^{* * * *}$, José Antonio Casajús ${ }^{* * * *}$, Lino Álvarez-Granda****, Cipriano Romero \\ Cerezo*, Pablo Tercedor* y Manuel Delgado-Fernández*
}

*Universidad de Granada, ${ }^{* *}$ Universidad de Málaga, ${ }^{* * *}$ Instituto de Ciencia y Tecnología de los Alimentos y Nutrición (Madrid) ${ }^{* * * *}$ Universidad de Zaragoza, *****Universidad de Cantabria

Resumen: A pesar de los reconocidos beneficios físicos, psíquicos y sociales que la práctica de actividad físico-deportiva produce en la salud de los jóvenes, la prevalencia de abandono y falta de este tipo de actividades está mostrando un importante aumento a nivel mundial, particularmente en los países desarrollados. Se precisan actuaciones educativas inmediatas para frenar esta situación. El objetivo del estudio es analizar la expresión de interés hacia la actividad físico-deportiva atendiendo al nivel de participación de ésta, y conocer los motivos de abandono y no práctica en una muestra representativa de adolescentes espańoles. Se efectuó un estudio descriptivo de corte transversal y se empleó la encuesta sobre comportamientos, actitudes y valores sobre actividad físico-deportiva del estudio AVENA (Alimentación y Valoración del Estado Nutricional en Adolescentes). La muestra fue de 2.859 adolescentes españoles (1.357 hombres, 1.502 mujeres; rango de edad: 13-18,5 años) y elegida de entre los escolares de Enseñanza Secundaria, procedentes tanto de centros públicos como privados de cinco ciudades espańolas: Granada, Madrid, Murcia, Santander y Zaragoza. Los principales resultados verifican una elevada expresión de interés hacia la práctica físico-deportiva aunque casi la mitad de los adolescentes indican un nivel de participación insuficiente respecto a lo que realmente querrían practicar. La exigencia del estudio y la pereza y desgana suponen los principales motivos de abandono de la misma. Respecto a los motivos de no práctica, la falta de tiempo, seguido muy de lejos por la pereza, la falta de gusto hacia la misma y el cansancio por el estudio o trabajo. El género y la edad modifican significativamente la expresión de interés y los motivos de abandono y no práctica físico-deportiva. El conocimiento de estos motivos podría permitir desarrollar programas de intervención eficaces y duraderos en la adopción de estilos de vida saludables en los jóvenes. Palabras clave: motivos, abandono de práctica deportiva, actividad física, deporte, salud, adolescente

Abstract: Despite the recognized physical, psychological and social benefits of physical and sport practice in the health of young people, prevalence of doprout and lack of such activities is increasing in developed countries. Interventions of different society institutions are required, being particularly relevant educatio-

Dirección de contacto: Alejandro César Martínez Baena

Departamento de Educación Física y Deportiva

Facultad de Ciencias de la Actividad Física y el Deporte. Carretera de Alfacar, S/N. Código Postal 18011. Correo electrónico: acmartinez@ugr.es nal institutions. The aim of the study is to analyze the interest physical activity and sport regarding the level of participation in this practice and to know the reasons for abandoning and non practicing in a representative sample of Spanish adolescents. A descriptive cross-sectional study was implemented and the survey about behaviours, attitudes and values related to physical activity and sport practice, which was developed in the AVENA (Feeding and assessment of nutritional status of spanish adolescents) study. The sample size was 2859 Spanish adolescents (1.357 men, 1.502 women; age range: $13-18.5$ years) and chosen from among students of secondary education, both from public and private schools in five Spanish cities: Granada, Madrid, Murcia, Santander and Zaragoza. The main results show a high level of interest about physical activity and sport practice although almost half of the adolescents show an insufficient level regarding the level that they would like to practice. The demanding school and laziness and reluctante are the main reasons for stop practicing. The reasons for no practicing are lack of time, and much further the laziness, lack of pleasure with the practice and tiredness because of the school or work. Gender and age modify significantly the interest and reasons for abandoning and non practicing physical activity and sports. The knowledge of these reasons could allow to develop effective and long-term successful intervention programmes for adopting healthy lifestyles in young people.

Keywords: reasons, dropping out of sports and organized physical activities, physical activity, sport, health, adolescent.

Resumo: Apesar dos reconhecidos benefícios físicos, psíquicos e sociais que produz a pratica de atividade fisico-esportivo em jovens, a prevalência de abandono e a falta dessa atividade esta crescendo a nível mundial especialmente em países desenvolvidos. Necessitam atuaçōes educativas imediatas para desacelerar essa situação. O objetivo desse estudo é de analisar a expressão do interesse à pratica físico-esportiva em relação com o nível de participaçáo e conhecer os motivos de desistência e a náo pratica em uma mostra representativa de adolescentes espanhóis. Foi realizado um estudo descritivo de corte transversal e empregou-se um questionário sobre comportamentos, atitudes e valores sobre a atividade físico-esportiva do estudo AVENA (alimentação e avaliação do estado nutricional em adolescentes). A mostra foi de 2.859 adolescentes espanhóis (1.357 homens, 1.502 mulheres; rango de idade: 13-18,5 anos) escolhida de centros escolares públicos e particulares do ensino secundário de cinco cidades espanholas: Granada, Madri, Murcia, Santander e Zaragoza. Os principais resultados verificam uma elevada expressão de interesse á pratica físicoesportiva, ainda que quase a metade dos adolescentes apresentam um nível de 
participação insuficiente a respeito do que realmente gostariam de praticar. A exigência dos estudos e a preguiça são os principais motivos de desistência da pratica de exercícios físicos. Em relação aos motivos da não pratica estão a falta de tempo, a preguiça, falta de gosto, cansaço por causa dos estudos e o trabalho. $\mathrm{O}$ gênero e a idade modificam significativamente a expressão do interesse e os motivos de desistência e da não pratica físico-esportiva. $\mathrm{O}$ conhecimento de esses motivos poderia permitir a elaboração de um programa de intervenções eficazes e de duração no estilo de vida saudável dos jovens.

Palavras chave: motivos, desistência da pratica esportiva, atividade física, esporte, saúde, adolescência.

\section{Introducción}

A pesar de la reconocida mejora que la práctica físico-deportiva regular produce en la calidad de vida a lo largo de los años (Blair y Morris, 2009), diversos estudios verifican un descenso progresivo de la práctica físico-deportiva con la edad en la población joven (Varo et al., 2003), demostrando una mayor tasa de abandono entre los 12 y los 18 ańos (Chillón et al., 2009).

Recientemente se ha observado mediante valoración objetiva (acelerometría) de la actividad física en jóvenes niveles de práctica bajos (Hagstromer et al., 2008), al no cumplir estos grupos de edad con la pauta recomendada de 60 minutos o más de actividad físico-deportiva diaria con una intensidad de moderada a vigorosa (Moller, Kristensen, Wedderkopp, Andersen y Floberg, 2009).

Diversos estudios constatan la existencia de estas fuertes tasas de abandono durante la adolescencia (Bodson 1997, Torre, 1998, Pierón, Telama, Almond y Carreiro da Costa, 1999; García Ferrando, 2001; Tercedor y cols., 2003; Palou, Ponseti, Gili, Borras y Vidal, 2005; Ruiz Juan, García Montes y Gómez López, 2005). Un abandono que queda muy bien explicado en el trabajo de Carlin, Salguero, Márquez y Garcés (2009), donde se fundamentan de manera clara las diversas tipologías del mismo de acuerdo a la decisión controlable o incontrolable de desertar de la práctica.

La práctica físico-deportiva tal cual está planteada, no responde a los intereses y motivaciones de este grupo de población (Moreno, Alonso, Martínez Galindo y Cervelló, 2005) y que habría que atender al análisis de dichos factores para analizar las causas de abandono y mantenimiento de actividad físico-deportiva (Moreno, Gómez y Pérez, 2007; Quevedo-Blasco, Quevedo-Blasco y Bermúdez, 2009) y las posibilidades de incremento de las mismas para la puesta en práctica de un estilo de vida saludable en los adolescentes (Cecchini et al., 2008).

Diversos estudios ya han analizado los motivos que alientan a estos jóvenes, pertenecientes tanto al ámbito escolar (Moreno, Zomeño, Marín, Cervello y Ruiz, 2009; Cecchini, Méndez y Muñiz, 2002) como al universitario (Moreno, Pavón, Gutiérrez y Sicilia, 2005; Pavón, Moreno, Gutiérrez y Sicilia, 2004), a practicar actividad físico-deportiva en su tiempo libre. Algunos de ellos, mediante la creación de sofisticadas herramientas (Moreno, Gonzalez-Cutre y Chillón, 2009), con el fin de disminuir los elevados índices de abandono en esta etapa adolescente (Cervelló, Escartí y Guzmán, 2007).

No obstante, resulta interesante conocer los motivos de abandono y no práctica de estos adolescentes con el fin de intentar reconvertir estos elementos en inductores, facilitadores y fortalecedores de práctica de actividad físico-deportiva para la adquisición de un estilo de vida activo y su conservación a lo largo del ciclo vital (Moreno et al., 2005).

Sobre motivos de abandono de práctica físico-deportiva en este grupo de población, son muchos los estudios que evidencian que la falta de tiempo aducida por los estudios y el aburrimiento o pereza por la actividad constituyen los elementos de deserción más destacables en estos chicos y chicas (Piéron, 2002; Ispizua, 2003). En un estudio realizado por Arribas Galárraga y Arruza Gabilondo (2004), se destacaron como principales causas de abandono de práctica de actividad físico-deportiva en estos adolescentes, el aburrimiento y el desarme del equipo de pertenencia. Más recientemente, en un estudio realizado por Macarro, Romero y Torres (2010), sobre 857 adolescentes pertenecientes a primer curso de Bachillerato en centros educativos públicos, concertados y privados de la provincia de Granada, se evidenció que un porcentaje relativamente elevado de los encuestados indicaba haber abandonado la práctica, siendo este porcentaje bastante superior en las chicas. El motivo más aludido para ese abandono resultó ser la falta de tiempo seguido muy de cerca por la preferencia en la realización de otras actividades de tiempo libre, así como la pereza o desgana. Respecto a los motivos de no práctica, tanto en chicos como en chicas, queda claro en diversos estudios (Escudero, Serra y Servera, 1992; Ponseti, 1998), que la falta de tiempo, seguido muy de cerca por la preferencia por otras actividades, destacan como causas de mayor peso para que se produzca el abandono.

Por tanto, resulta relevante seguir investigando en los motivos por los que no se practica o se abandona la actividad físico-deportiva en la etapa adolescente, como ha sido parcialmente tratado en España en estos estudios. En este caso, sobre una muestra representativa de adolescentes pertenecientes al ámbito nacional.

De esta forma, el objetivo de la presente investigación es analizar la expresión de interés hacia la actividad físico-deportiva atendiendo al nivel de participación de ésta y conocer los motivos abandono y no práctica en una muestra representativa de adolescentes españoles. 


\section{Método}

\section{Participantes}

El presente trabajo forma parte del Estudio AVENA (Alimentación y Valoración del Estado Nutricional en Adolescentes), cuya metodología completa ha sido publicada con anterioridad (González-Gross et al., 2003). Para la determinación del tamaño total de muestra se tomó el parámetro de mayor varianza en la población, utilizando los datos que había publicados en la bibliografía cuando se planeó el estudio (Moreno et al, 1997): el índice de masa corporal (IMC). El muestreo estuvo determinado por esta dispersión. El nivel de confianza es del $95 \%$ con un error $\pm 0,25$. Se calculó un $\mathrm{n}=2.100$ para el estudio completo. La muestra se sobredimensionó para prevenir pérdidas de información obteniendo una muestra de 2.859 sujetos (1.357 hombres, 1.502 mujeres), con edades comprendidas entre los 12,40 y los 18,70 años y una media de 15,37 \pm 1,44 años. Los sujetos fueron elegidos de entre los escolares de Enseñanza Secundaria o Formación Profesional, procedentes tanto de centros públicos como privados de cinco ciudades españolas: Granada, Madrid, Murcia, Santander y Zaragoza. El 98,1\% de los sujetos era de raza caucasiana, un $1,1 \%$ de latinoamericanos indios y el $0,8 \%$ restante de raza afroamericana, europeos del este, África subsahariana, África negra, asiática y gitana.

Para obtener grupos de edad equivalentes, la edad fue agrupada en cinco bloques desde los 13 a los 17-18 años. En el grupo de 13 años se incluyen los sujetos desde 12,40 hasta 13,99 años, y así sucesivamente. Se ajustó finalmente con un factor de ponderación para equilibrar la muestra según la distribución de la población española y garantizar la representación real de cada uno de los grupos definidos por los dos factores mencionados (Fuente: Instituto Nacional de Estadística). Sobre la muestra inicial un total de 2.210 sujetos (1136 varones y 1074 mujeres) informaron de su interés hacia la práctica físico-deportiva, 2194 (1134 varones y 1060 mujeres) respecto a su nivel de participación en relación a dicho interés, 609 (221 varones y 388 mujeres) sobre los motivos de abandono de práctica y 605 (185 varones y 420 mujeres) sobre los motivos de no practicar actividad físico-deportiva.

\section{Instrumento}

A pesar de la existencia de herramientas ya validadas y empleadas de manera habitual en estudios con propósito similar al de este trabajo (Martínez-Gómez et al., 2009; Moreno et al., 2009; Quevedo-Blasco, Quevedo-Blasco y Bermúdez, 2009; Moreno, Cervelló y Martínez, 2007), se utilizó como herramienta de toma de datos un cuestionario elaborado específicamente en el estudio Avena, ya propuesto en anteriores trabajos (Tercedor et al., 2003; Vicente-Rodríguez, Rey-Ló- pez, Martín-Matillas, Moreno, Wärnberg y Redondo, 2008; Chillón et al., 2009; Ortega et al., 2010).

Dicho instrumento es construido atendiendo a una síntesis realizada a partir de herramientas ya utilizadas en la bibliografía, aportaciones del propio grupo de investigación y estudios experimentales realizados por los propios investigadores del proyecto (González-Gross et al., 2003). Del mismo emergen las siguientes categorías:

Actitudes, intereses y valores hacia la actividad física y el deporte, mediante los items correspondientes de la encuesta de García Ferrando (1997).

Motivaciones y causas de abandono hacia la práctica de actividad físico-deportiva, mediante la misma técnica y los items del cuestionario de Mendoza, Sagrera y Batista (1994).

Actitudes hacia el proceso y el producto relacionadas con la salud, a partir del cuestionario de actitudes de actividad física orientada a la salud de Pérez Samaniego (1999).

Percepción de la utilidad de la práctica de actividad física y el deporte hacia la salud, valorada a partir del diferencial semántico de Sánchez Bañuelos (1996).

De dicho cuestionario se utilizaron las siguientes preguntas:

- Considerando la actividad físico-deportiva en todas sus formas, es decir, como juego, espectáculo, ejercicio físico y diversión, e independientemente de que la practiques o no, ¿dirías que te interesa? (1-Mucho, 2-Bastante, 3-Poco, 4-Nada).

- Respecto al interés por la actividad físico-deportiva y a su nivel de práctica, se pueden considerar seis grupos de población, ¿̇con cuál te identificas más? (1- Me interesa la actividad físico-deportiva y la practico suficientemente, 2- Me interesa la actividad físico-deportiva, pero no la practico tanto como quisiera, 3- No me interesa la actividad físico-deportiva, pero la practico por obligación (enfermedad, clases, trabajo, etc.), 4- Me interesa la actividad físico-deportiva, la he practicado, pero ahora ya no la practico, 5- Nunca he practicado actividad físico-deportiva, pero me gustaría hacerlo, 6No la practico ni me interesa).

- Si has dejado o abandonado la práctica deportiva, de los motivos que aparecen a continuación, dinos, por orden de importancia, los que más influyeron en su decisión (máximo dos respuestas; 1- No tenía instalaciones deportivas adecuadas, 2- No tenía instalaciones deportivas cerca, 3- Por lesiones, 4- Por salud, 5- Me enfadé con el entrenador, 6- Me enfadé con los directivos, 7- Mis padres no me dejaban, 8- No le gustaba a mi novio/a, 9Los estudios me exigían demasiado, 10- Salía muy cansado o muy tarde del trabajo, 11- Por pereza y desgana, 12- Dejó de gustarme hacer actividad físico-deportiva, 13- No le veía los beneficios, 14- Mis amigos no hacían actividad físico-deportiva, 15- Falta de apoyo y estímulo, 16- Por falta de dinero, 17- Otra, ¿cuál?). 
- Si no practicas actividad físico-deportiva en la actualidad, dime, por favor, las dos razones principales por orden de importancia (1- No me gusta, 2- Por salud, 3No me enseñaron en la escuela, 4- No le veo beneficios, 5- No le veo utilidad, 6- No tengo tiempo, 7- No hay instalaciones deportivas cerca, 8- No hay instalaciones deportivas adecuadas, 9- Salgo muy cansado del trabajo o del estudio, 10- Por pereza y desgana, 11- Otra, ¡cuál?).

\section{Procedimiento}

La aplicación del cuestionario se realizó de forma negociada con los centros y con la autorización de los directores de los mismos, así como con el consentimiento de los padres. Los cuestionarios fueron autoadministrados a los alumnos a primera hora de la mańana y dentro de su aula en el horario habitual de clases. Los encuestadores siguieron un guión unificado para dar las instrucciones oportunas. Previamente se les indicó a los alumnos el objeto de estudio y se les pidió que contestasen con seriedad y máxima sinceridad, asegurándoles la confidencialidad de las respuestas.

\section{Análisis estadístico}

Los resultados descriptivos, al ser variables nominales, se es- tudiaron y se presentan en frecuencias y porcentajes.

El análisis inferencial se inició estudiando la normalidad de las variables mediante la prueba de Kolmogorov-Smirnov, observándose una distribución no normal de los datos; además, la prueba de Levene resultó en varianzas desiguales. Por tanto, se aplicaron técnicas no paramétricas para comprobar las diferencias entre dos grupos independientes chicos-chicas (empleando el test de Mann-Whitney) y las diferencias entre grupos de edad (empleando el test de Kruskal-Wallis). En ambos casos se ha exigido un valor de significación de $\mathrm{p}<$ 0.05. Para todos los cálculos del análisis de resultados se utilizó el paquete estadístico SPSS v. 15.

\section{Resultados}

En el análisis del grado de interés de los jóvenes respecto a la práctica físico-deportiva diferenciado por género, se evidencian diferencias significativas $(\mathrm{p}<0.01)$. En la tabla 1 , observamos que el $56,7 \%$ de los chicos y el $34,8 \%$ de las chicas muestran "mucho" interés hacia este tipo de prácticas. No obstante, ambos reflejan un interés global "de mucho a bastante" elevado ( $93,1 \%$ de chicos y $82,8 \%$ de chicas). En cambio, no se evidencian diferencias significativas respecto a dicha variable en los diversos intervalos de edad analizados.

Tabla 1. Prevalencia del interés hacia la práctica físico-deportiva estratificado por género y edad

\begin{tabular}{|c|c|c|c|c|c|c|c|c|c|}
\hline \multirow[b]{2}{*}{ Interés } & \multirow[t]{2}{*}{ Total (n) } & \multicolumn{2}{|c|}{ Género (\%) } & \multicolumn{6}{|c|}{ Edad (\%) } \\
\hline & & $\begin{array}{l}\text { Chico } \\
\mathrm{n}=1136\end{array}$ & $\begin{array}{c}\text { Chica } \\
\mathrm{n}=1074\end{array}$ & $\begin{array}{c}13 \\
\mathrm{n}=393\end{array}$ & $\begin{array}{c}14 \\
\mathrm{n}=421\end{array}$ & $\begin{array}{c}15 \\
\mathrm{n}=493\end{array}$ & $\begin{array}{c}16 \\
\mathrm{n}=430\end{array}$ & & $\begin{array}{c}17-18 \\
n=474\end{array}$ \\
\hline Mucho & 1028 & 56,7 & 34,8 & 49,4 & 46,1 & 49,1 & 45,8 & 41,6 & \\
\hline Bastante & 908 & 34,6 & 48 & 41,2 & 40,1 & 38,1 & 42,1 & 43,6 & \\
\hline Poco & 248 & 6,7 & 16 & 7,9 & 12,6 & 12 & 10,7 & 13,7 & \\
\hline Nada & 26 & 1,1 & 1,2 & 1,5 & 1,2 & 0,8 & 1,4 & 1,1 & \\
\hline & & $P$ género $=0$ & & P edad $=0$ & & & & & \\
\hline
\end{tabular}

Con respecto al interés por la actividad físico-deportiva unido al nivel de práctica, observamos diferencias significativas para género y edad $(\mathrm{p}<0.001$ ) (Tabla 2). El $51,1 \%$ de los chicos frente al 21,8\% de las chicas señalan un alto interés unido a una práctica suficiente. En cambio, son las chicas las que a pesar de contar con una buena intención, indican no practicarla como quisieran $(45,1 \%)$, frente a un $34,7 \%$ de los chicos. Destacar también que el 23,1\% de las jóvenes seńalan una práctica por obligación a pesar de su bajo interés, con- trastado con un $9,5 \%$ de los chicos.

Se observan diferencias en los intervalos de edad analizados. Así, en el enunciado "Me interesa y la practico suficientemente", se pasa de un porcentaje del $47,4 \%$ a los 13 ańos hasta el 25,5\% de los 17-18 ańos. En cambio, en el enunciado "No me interesa, pero practico por obligación", se pasa de un $30,7 \%$ a un $9,5 \%$ a los 13 años de práctica por obligación a los 17-18 años. 
Tabla 2. Diferencias por género y edad respecto al interés por la actividad físico-deportiva y a su nivel de práctica

\begin{tabular}{|c|c|c|c|c|c|c|c|c|}
\hline \multirow[b]{2}{*}{ Interés + nivel } & \multirow[t]{2}{*}{ Total (n) } & \multicolumn{2}{|c|}{ Género (\%) } & \multicolumn{5}{|c|}{ Edad (\%) } \\
\hline & & $\begin{array}{c}\text { Chico } \\
\mathrm{n}=1134\end{array}$ & $\begin{array}{c}\text { Chica } \\
\mathrm{n}=1060\end{array}$ & $\begin{array}{c}13 \\
\mathrm{n}=388\end{array}$ & $\begin{array}{c}14 \\
\mathrm{n}=420\end{array}$ & $\begin{array}{c}15 \\
\mathrm{n}=490\end{array}$ & $\begin{array}{c}16 \\
\mathrm{n}=426\end{array}$ & $\begin{array}{c}17-18 \\
\mathrm{n}=468\end{array}$ \\
\hline $\begin{array}{l}\text { Me interesa y la practico } \\
\text { suficientemente }\end{array}$ & 810 & 51,1 & 21,8 & 47,4 & 40,7 & 39,2 & 33,6 & 25,55 \\
\hline $\begin{array}{l}\text { Me interesa, pero no practi- } \\
\text { co como quisiera }\end{array}$ & 871 & 34,7 & 45,1 & 35,6 & 41,4 & 39,6 & 40,6 & 35,8 \\
\hline $\begin{array}{l}\text { No me interesa, pero practi- } \\
\text { co por obligación }\end{array}$ & 76 & 2,6 & 4,4 & 4,1 & 2,4 & 4,7 & 3,5 & 2,7 \\
\hline $\begin{array}{l}\text { Me interesa, la he practi- } \\
\text { cado, pero ahora ya no la } \\
\text { practico }\end{array}$ & 353 & 9,5 & 23,1 & 9,5 & 11,7 & 13,1 & 18,8 & 30,7 \\
\hline $\begin{array}{l}\text { Nunca he practicado, pero } \\
\text { me gustaría hacerlo }\end{array}$ & 43 & 0,7 & 3,3 & 2,6 & 1,9 & 2,4 & 1,6 & 2,3 \\
\hline \multirow[t]{2}{*}{$\begin{array}{l}\text { No la practico ni me } \\
\text { interesa }\end{array}$} & 41 & 1,5 & 2,3 & 0,8 & 1,9 & 1 & 1,9 & 2,95 \\
\hline & \multicolumn{3}{|c|}{$P$ género $=0,000$} & \multicolumn{5}{|c|}{ P edad $=\mathbf{0 , 0 0 0}$} \\
\hline
\end{tabular}

En la comparación respecto a los motivos de abandono de práctica físico-deportiva, se observan diferencias significativas para género $(\mathrm{P}=0.002)$ y edad $(\mathrm{P}<0.001)$ (tabla 3$)$. El principal motivo de abandono resulta ser el estudio, siendo el porcentaje algo más alto en las chicas $(36,1 \%)$ frente al 26,2\% de los chicos. El segundo motivo, la pereza, con un $15,7 \%$ en ellas y un $14,5 \%$ en ellos.

Respecto a la edad, se aprecia un aumento en el abandono por estudios, pasando de un 23,7\% a los 13 ańos hasta un $42,05 \%$ a los 17-18 años. De igual forma, la pereza, aumenta de un $11,8 \%$ a los 13 ańos a un $20,75 \%$ a los $17-18$ como motivo de abandono de la práctica.

Tabla 3. Diferencias por género y edad respecto a los motivos de abandono de práctica físico-deportiva

\begin{tabular}{|c|c|c|c|c|c|c|c|c|}
\hline \multirow[b]{2}{*}{ Motivos abandono } & \multirow[t]{2}{*}{ Total (n) } & \multicolumn{2}{|c|}{ Género (\%) } & \multicolumn{5}{|c|}{ Edad (\%) } \\
\hline & & $\begin{array}{l}\text { Chico } \\
\mathrm{n}=221\end{array}$ & $\begin{array}{l}\text { Chica } \\
\mathrm{n}=388\end{array}$ & $\begin{array}{c}13 \\
\mathrm{n}=76\end{array}$ & $\begin{array}{c}14 \\
\mathrm{n}=103 \\
\end{array}$ & $\begin{array}{c}15 \\
\mathrm{n}=129\end{array}$ & $\begin{array}{c}16 \\
\mathrm{n}=120\end{array}$ & $\begin{array}{c}17-18 \\
\mathrm{n}=185\end{array}$ \\
\hline No tenía instalaciones adecuadas & 28 & 7,7 & 2,8 & 5,3 & 5,8 & 7 & 4,2 & 3,15 \\
\hline No tenia instalaciones cerca & 45 & 7,7 & 7,2 & 10,5 & 10,7 & 6,2 & 6,7 & 4,4 \\
\hline Por lesiones & 57 & 13,6 & 7,2 & 5,3 & 9,7 & 7 & 11,7 & 8,35 \\
\hline Por salud & 23 & 4,1 & 3,9 & 9,2 & 3,9 & 5,4 & 1,7 & 3,4 \\
\hline Enfado con entrenador & 32 & 7,7 & 3,9 & 10,5 & 5,8 & 5,4 & 4,2 & 4,5 \\
\hline Enfado con directivos & 3 & 0,9 & 0,3 & 0 & 1 & 0 & 1,7 & 0 \\
\hline Mis padres no me dejaban & 13 & 1,8 & 2,3 & 3,9 & 0 & 3,1 & 2,5 & 1,1 \\
\hline Por mi novio/a & 2 & 0 & 0,5 & 1,3 & 1 & 0 & 0 & 0 \\
\hline Por estudios & 199 & 26,2 & 36,1 & 23,7 & 24,3 & 31,8 & 31,7 & 42,05 \\
\hline Por trabajo & 17 & 2,7 & 3,1 & 2,6 & 3,9 & 4,7 & 4,2 & 0,35 \\
\hline Por pereza & 93 & 14,5 & 15,7 & 11,8 & 15,5 & 10,1 & 15 & 20,75 \\
\hline Dejo de gustarme & 25 & 3,6 & 4,4 & 5,3 & 5,8 & 3,9 & 2,5 & 4,15 \\
\hline No le veía beneficios & 8 & 1,4 & 1,3 & 3,9 & 1 & 1,6 & 1,7 & 0 \\
\hline Mis amigos no hacían & 10 & 2,7 & 1 & 2,6 & 1,9 & 4,7 & 0 & 0 \\
\hline Falta de apoyo y estímulo & 14 & 1,4 & 2,6 & 1,3 & 0 & 3,1 & 4,2 & 2,1 \\
\hline Por falta de dinero & 11 & 1,8 & 1,5 & 1,3 & 3,9 & 0,8 & 1,7 & 2,4 \\
\hline \multirow[t]{2}{*}{ Otra } & 29 & 2,3 & 6,2 & 1,3 & 5,8 & 5,4 & 6,7 & 3,2 \\
\hline & \multicolumn{3}{|c|}{$P$ género $=0,002$} & \multicolumn{5}{|c|}{$P$ edad $=0,000$} \\
\hline
\end{tabular}

Respecto a los motivos de no práctica físico-deportiva, se observan diferencias significativas para género $(\mathrm{P}<0.001)$ y edad $(\mathrm{P}<0.001)$ (tabla 4). El principal motivo de no práctica resulta ser la falta de tiempo, siendo el porcentaje algo más 
alto en las chicas (56\%) frente al 44,3\% de los chicos. El segundo motivo, vuelve a ser nuevamente la pereza, con un $14,6 \%$ en ellos y un $9,8 \%$ en ellas.

También se observan discordancias en los intervalos de edad analizados en cuanto a estos dos motivos. Así, podemos apreciar un aumento con la edad de no práctica por falta de tiempo, pasando de un $48,1 \%$ a los 13 años hasta un 59,75\% a los 17-18. De igual forma, se produce un aumento por pereza pasando de un 3,8\% a los 13 ańos a un $12,1 \%$ a los $17-18$ años.

Tabla 4. Diferencias por género y edad respecto a los motivos de no práctica físico-deportiva

\begin{tabular}{|c|c|c|c|c|c|c|c|c|}
\hline \multirow[b]{2}{*}{ Motivos no práctica } & \multirow[t]{2}{*}{ Total (n) } & \multicolumn{2}{|c|}{ Género (\%) } & \multicolumn{5}{|c|}{ Edad (\%) } \\
\hline & & $\begin{array}{l}\text { Chico } \\
\mathrm{n}=185\end{array}$ & $\begin{array}{l}\text { Chica } \\
\mathrm{n}=420 \\
\end{array}$ & $\begin{array}{c}13 \\
\mathrm{n}=79\end{array}$ & $\begin{array}{c}14 \\
\mathrm{n}=96\end{array}$ & $\begin{array}{c}15 \\
\mathrm{n}=116\end{array}$ & $\begin{array}{c}16 \\
\mathrm{n}=125\end{array}$ & $\begin{array}{l}17-18 \\
\mathrm{n}=185\end{array}$ \\
\hline No me gusta & 53 & 11,4 & 7,6 & 13,9 & 7,3 & 9,5 & 6,4 & 9,55 \\
\hline Por salud & 27 & 5,4 & 4 & 6,3 & 4,2 & 4,3 & 4 & 5,35 \\
\hline No me enseñaron en la escuela & 2 & 0 & 0,5 & 1,3 & 0 & 0 & 0 & 0,35 \\
\hline No le veo beneficios & 9 & 2,2 & 1,2 & 1,3 & 0 & 1,7 & 4 & 0,35 \\
\hline No le veo utilidad & 7 & 1,6 & 1 & 0 & 1 & 1,7 & 1,6 & 0,75 \\
\hline No tengo tiempo & 317 & 44,3 & 56 & 48,1 & 52,1 & 45,7 & 52,8 & 59,75 \\
\hline $\begin{array}{l}\text { No hay instalaciones deportivas } \\
\text { cerca }\end{array}$ & 34 & 6,5 & 5,2 & 12,7 & 6,3 & 8,6 & 4 & 1,1 \\
\hline $\begin{array}{l}\text { No hay instalaciones deportivas } \\
\text { adecuadas }\end{array}$ & 9 & 1,6 & 1,4 & 1,3 & 2,1 & 1,7 & 0 & 1,8 \\
\hline $\begin{array}{l}\text { Salgo muy cansado del trabajo o } \\
\text { estudio }\end{array}$ & 53 & 8,6 & 8,8 & 10,1 & 9,4 & 8,6 & 9,6 & 5,75 \\
\hline Por pereza & 68 & 14,6 & 9,8 & 3,8 & 13,5 & 12,1 & 12 & 12,1 \\
\hline \multirow[t]{2}{*}{ Otras } & 26 & 3,8 & 4,5 & 1,3 & 4,2 & 6 & 5,6 & 3,25 \\
\hline & \multicolumn{3}{|c|}{$P$ género $=0,000$} & \multicolumn{5}{|c|}{$P$ edad $=0,000$} \\
\hline
\end{tabular}

\section{Discusión y Conclusiones}

Los resultados de este estudio realizado sobre una muestra representativa de adolescentes espańoles, verifican una elevada expresión de interés hacia la práctica físico-deportiva aunque casi la mitad de los adolescentes indican un nivel de participación insuficiente respecto a lo que realmente querrían practicar. La exigencia de los estudios y la pereza suponen los principales motivos de abandono de la misma. En cuanto a los motivos de no práctica, la falta de tiempo, seguido muy de lejos por la pereza, la falta de gusto hacia la misma y el cansancio por el estudio o trabajo, destacan como las grandes razones expuestas. El género y la edad modifican significativamente la expresión de interés y los motivos de abandono y no práctica físico-deportiva.

Los hallazgos de nuestro estudio muestran una elevada expresión de interés de los adolescentes españoles respecto a la práctica físico-deportiva, siendo éste mayor en chicos, si bien esta expresión de interés disminuye con la edad. Además, los resultados del estudio de Lorenzo y Ayllón (2002) verifican que efectivamente son los chicos los que presentan una mayor expresión de interés. En este sentido, resulta importante destacar el hecho de que son las chicas las que a pesar de contar con una buena predisposición, indican no practicar como quisieran. Anteriores estudios (Cecchini, González, Carmo- na y Contreras, 2004; Sarrazin, Vallerand, Guillet, Pelletier y Cury, 2002) revelaron que la disminución progresiva del interés está directamente relacionada con el abandono de dicha práctica (Nuviala y Nuviala, 2005). Este descenso de la práctica físico-deportiva de manera paralela al aumento de la edad, se explican por una disminución progresiva del interés y la motivación unida al desarrollo de estos jóvenes (Pavón y Moreno, 2008).

En referencia a los motivos de abandono y de no práctica físico-deportiva, destacan la falta de tiempo por estudios y la pereza como principales causas, con una mayor incidencia en las chicas que en los chicos y con un incremento de ambos con la edad. Estos resultados son semejantes a los observados en estudios anteriores (Boiche y Sarrazin, 2009; De Hoyo y Sañudo, 2007; García Ferrando, 2001). Considerando los resultados de nuestro estudio en general, los jóvenes adolescentes que manifiestan motivos de carácter extrínseco, tales como la falta de tiempo por la exigencia del estudio o la falta de instalaciones, podrían catalogarse como adolescentes interesados por la práctica, pero de poca participación a pesar de su buena predisposición, concordando con los resultados de Cecchini, Méndez y Contreras (2005). Lo que Cervelló (2002) denomina como abandono asociado a causas incontrolables, aunque la gestión del uso del tiempo se podría modificar. Por el contrario, los jóvenes que alegan motivos 
de carácter intrínseco, como la pereza, podrían relacionarse con una disminución en el interés y el gusto por la práctica físico-deportiva. En palabras de Sarrazin y Guillet (2001), no práctica por descontento, identificaría a jóvenes no satisfechos en sus necesidades y, por lo tanto, con pocas ganas de implicación. En general y en la línea de estudios como el de Cecchini y González (2008), entendemos que tanto los motivos de carácter extrínseco (falta de tiempo por exigencia de los estudios) como los motivos de carácter intrínseco (pereza o desgana), condicionan los niveles de práctica y tienen importancia en el abandono o no de práctica de los adolescentes (Mason, Mathias y Skevington, 2008).

Desde nuestra óptica, resulta importante incidir en una mejor gestión deportiva para la dotación de mayor cantidad de instalaciones y una reeducación escolar en estos chicos para una mejor organización de su tiempo libre. La labor del docente de Educación Física se presenta crucial, como ha sido reflejado en estudios previos (Moreno, Hellín, Hellín, Cervelló y Sicilia, 2008; Penney y Chandler, 2000). Para solucionar el problema de carácter intrínseco como la pereza quizá sería necesario un mayor trabajo por parte de los agentes de la comunidad educativa, mediante estrategias de intervención más cercanas a las expectativas, intereses y demandas sociales y juveniles (Prat y Gómez, 2009; Pérez y Delgado, 2007).

El actual trabajo presenta la limitación de ser una primera aproximación las creencias y actitudes de los adolescentes españoles respecto a la conducta de actividad física, y por tanto, el análisis estadístico es básico y fundamentalmente descriptivo. Por otra parte, el tamańo muestral y la representatividad de la población espańola en los sujetos participantes son puntos fuertes que proporcionan rigor y la posibilidad de transferencia social de dicho trabajo.

En conclusión, los hallazgos de este trabajo muestran una elevada expresión de interés de los adolescentes españoles hacia la práctica físico-deportiva que no se acompaña del mismo nivel de participantes, siendo la exigencia de los estudios y la pereza los principales motivos de abandono de la misma. El género y la edad modifican significativamente la expresión de interés y los motivos de abandono y no práctica físico-deportiva. Estos resultados pueden ayudar a construir estrategias de promoción de la salud a través de las cuales se conseguirá acrecentar estilos de vida más activos entre la población joven $y$, en consecuencia, la adherencia hacia actividades físicas para toda la vida, reduciendo los índices de abandono y un aumento de los beneficios incorporados a la práctica físicodeportiva continuada.

\section{Referencias bibliográficas}

Arribas Galárraga, S. y Arruza Gabilondo, A. (2004). El abandono de la práctica de la actividad física y el deporte en jóvenes escolares de Guipúzcoa. En J. Díaz (Coord.), III Congreso Nacional de Deporte en edad escolar (pp. 365-380). Dos Hermanas (Sevilla): Patronato Municipal de Deportes del Excmo. Ayuntamiento de Dos Hermanas.

Ballesteros-Arribas, J., Dal-re Saavedra, M., Pérez-Farinós, N. y VillarVillalba, C. (2007). La estrategia para la nutrición, actividad física y prevención de la obesidad: estrategia NAOS. Revista Española de Salud Publica, 81, 443-9.

Blair, S. y Morris, J. (2009). Healthy Hearts and the universal benefits of being physically active: physical activity and health. Annals of epidemio$\log y, 19(4), 253-256$

Bodson, D. (1997). La pratique du sport en communauté francaise. Sinthése analytique des résultats. Sport, 159, 5-42.

Boiche, J. y Sarrazin, P. (2009). Proximal and distal factors associated with dropout versus maintained participation in organized sport. Journal of sports science and medicine, 8(1), c-16.

Carlin, M., Salguero, A., Márquez, S. y Garcés, E. (2009). Análisis de los motivos de retirada de la práctica deportiva y su relación con la orientación motivacional en deportistas universitarios. Cuadernos de Psicología del Deporte, 9(1), 85-99.

Cecchini, J. y González, C. (2008). Motivos por los que los estudiantes universitarios no practican deporte, Revista Fuentes, 8, 1-10.

Cecchini, J., González, C., Carmona, A. y Contreras, O. (2004). Relaciones entre clima motivacional, la orientación de meta, la motivación intrínseca, la autoconfianza, la ansiedad y el estado de ánimo en jóvenes deportistas. Psicothema, 16(1), 104-109.

Cecchini, J., Gonzalez, C., Méndez, A., Fernández-Río, J., Contreras, O. y Romero, S. (2008). Metas sociales y de logro, persistencia-esfuerzo e intenciones de práctica deportiva en el alumnado de Educación Física. Psicothema, 20(2), 260-265.
Cecchini, J., Méndez, A. y Contreras, O. (2005). Motivos de abandono de la práctica del deporte juvenil. Cuenca: Ediciones de la Universidad de Castilla-La Mancha, $1^{\text {a }}$ Ed.

Cecchini, J.; Méndez, A.; Muñiz, J. (2002). Motivos de práctica deportiva en escolares españoles. Psicothema, 14, 523-531.

Cervelló, E. (2002). Abandono deportivo: Propuestas para favorecer la adherencia a la práctica deportiva. En J. Dosil (Ed.), Psicología y rendimiento deportivo, 175-187.

Cervelló, E., Escartí, A. y Guzmán, J. (2007). Youth sport dropout from the achievement goal theory. Psicothema, 19(1), 65-71.

Chillón, P., Ortega, F., Ruiz, J., Pérez, I., Martín-Matillas, M., Valtueña, J., et al. (2009). Socio-economic factors and active commuting to school in urban Spanish adolescents: the AVENA study. European Journal of Public Health, 19 (5), 470-476.

De Hoyo, M. y Sañudo, B. (2007). Motivos y hábitos de práctica de actividad física en escolares de 12 a 16 años en una población rural de Sevilla. Revista Internacional de Medicina y Ciencias de la Actividad Física y el Deporte, 7(26), 87-98.

Escudero, J., Serra, M. y Servera, M. (1992). Estudi dels hàbits esportius de la joventud de les Illes Balears. Palma: Conselleria de Cultura, Educació i Esports.

García Ferrando, M. (1997). Los españoles y el deporte, 1980-1995 (un estudio sociológico sobre comportamientos, actitudes y valores). Consejo Superior de Deportes (Madrid), Tirant lo blanch (Valencia).

García-Ferrando, M. (2001). Los españoles y el deporte: Prácticas y comportamientos en la última década del siglo XX. Encuesta sobre los hábitos deportivos de los españoles, 2000. Madrid: Ministerio de Educación, Cultura y Deporte. Consejo Superior de Deportes.

González-Gross, M., Castillo, M., Moreno, L., Nova, E., González-Lamuño, D., Pérez-Llamas, F., et al. (2003). Alimentación y valoración del estado nutricional de los adolescentes españoles (Proyecto AVENA). 
Evaluación de riesgos y propuesta de intervención I. Descripción metodológica del estudio. Nutr Hosp, 18, 15-28.

Hagstromer, M., Bergman, P., De Bourdeaudhuij, I., Ortega, F., Ruiz, J., Manios, Y., et al. (2008). Concurrent validity of a modified version of the International Physical Activity Questionnaire (IPAQ-A) in European adolescents: The HELENA Study. International journal of obesity, 32, 42-48.

Ispizua, M. (2003). Hábitos deportivos de la población de Bizkaia. Año 2001. Bilbao: Diputación Foral de Bizkaia.

Lorenzo, F. y Ayllón, F. (2002). Un estudio sobre intereses, actitudes y práctica de actividad físico-deportiva y género con población de educación secundaria Obligatoria. Revista Internacional de Medicina y Ciencias de la Actividad Fisica y el Deporte, 2(8), 253-277.

Macarro, J., Romero, C. y Torres, J. (2010). Motivos de abandono de la práctica de actividad físico-deportiva en los estudiantes de Bachillerato de la provincia de Granada. Revista de Educación, 343, 495-519.

Martínez-Gómez, D., Martínez-De-Haro, V., Del-Campo, J., Zapatera, B., Welk, G., Villagra, A.; et al. (2009). Validez de cuatro cuestionarios para valorar la actividad física en adolescentes espańoles. Gaceta Sanitaria, 23(6), 512-517.

Mason, V., Mathias, B. y Skevington, S. (2008). Accepting low back pain: is it related to a good quality of life?. Clinical Journal Pain, 24(1), 22-29.

Moller, N., Kristensen, P., Wedderkopp, N., Andersen, L. y Froberg, K. (2009). Objectively measured in 1997/1998 vs 2003/2004 in Danish children: The European Youth Heart Study. Scandinavian journal of medicine and science in sports, 19(1), 19-29.

Moreno, J., Alonso, N., Martínez Galindo, C. y Cervelló, E. (2005). Motivación, disciplina, coeducación y estado de flow en educación física: Diferencias según la satisfacción, la práctica deportiva y la frecuencia de práctica. Cuadernos de Psicología del Deporte, 5(1-2), 225-243.

Moreno, J., Cervelló, E. y Martínez, A. (2007). Validación de la Escala de Medida de los Motivos para la Actividad Física-Revisada en espańoles: Diferencias por motivos de participación. Anales de Psicología, 23(1), 167-176.

Moreno, L., Fleta, J., Mur, L., Feja, C., Sarría, A. y Bueno, M. (1997). Indices of body fat distribution in Spanish children aged 4.0 to 14.9 years. J Pediatr Gastroenterol Nutr, 25, 175-81.

Moreno, J., González-Cutre, D. y Chillón, M. (2009). Preliminary Validation in Spanish of a Scale Designed to Measure Motivation in Physical Education Classes: The Perceived Locus of Causality (PLOC) Scale. The Spanish Journal of Psychology, 12(1), 327-337.

Moreno, J., Hellín, P., Hellín, G., Cervelló, E. y Sicilia, A. (2008). Assessment of Motivation in Spanish Physical Education Students: Applying Achievement Goals and Self-Determination Theories. The Open Education Journal, 1, 15-22.

Moreno, J., Pavón, A., Gutiérrez, M. y Sicilia, A. (2005). Motivaciones de los universitarios hacia la práctica físico-deportiva. Revista Internacional de Medicina y Ciencias de la Actividad Física y el Deporte, 5(19), 154-165.

Moreno, J., Zomeño, T., Marín, L., Cervello, E. y Ruiz, L. (2009). Variables motivacionales relacionadas con la práctica deportiva extraescolar en estudiantes adolescentes de educación física. Apunts, 95, 38-43.

Nuviala, A. y Nuviala, R. (2005). Abandono y continuidad de la práctica deportiva escolar organizada desde la perspectiva de los técnicos de una comarca aragonesa. Revista Internacional de Medicina y Ciencias de la Actividad Física y el Deporte, 5(19), 295-307.

Ortega, F., Chillón, P., Ruiz, J., Albers, U., Álvarez-Granda, J., Marcos, A., et al. (2010). Sleep patterns in Spanish adolescents: associations with TV watching and leisure-time physical activity. Eur J Appl Physiol, 110(3), 563-573.

Pavón, A., Moreno, J., Gutiérrez, M. y Sicilia, A. (2004). Motivos de práctica físico-deportiva según la edad y el género en una muestra de universitarios. Apunts, 76, 13-21.

Pavón, A. y Moreno, J. (2008). Actitud de los universitarios ante la práctica físico-deportiva: diferencias por géneros. Revista de Psicología del Deporte, 17(1), 7-23.

Penney, D. y Chandler, T. (2000). Physical education: What future(s)? Sport Education and Society, 5(1), 71-87.

Pérez, I. y Delgado, M. (2007). Mejora de los conocimientos, procedimientos y actitudes del alumnado de secundaria tras un programa de intervención en educación física para la salud. Motricidad. European Journal of Human Movement, 18, 61-77.

Piéron, M. (2002). Estudi sobre els hàbits esportius dels escolars d'Andorra. Andorra: Govern d'Andorra.

Ponseti, X. (1998). Anàlisi de la pràctica esportiva dels joves de Mallorca en el segon cicle de l'ESO. Tesis doctoral no publicada.

Prat, M. y Gómez, I. (2009). Physical Education and social context: Influences and repercussions for curricular and educational proposals. Cultura y Educación, 21(1), 19-30.

Quevedo-Blasco, V., Quevedo-Blasco, R. y Bermúdez, M. (2009). Análisis de la motivación en la práctica de actividad físico-deportiva en adolescentes. Revista de Investigación en Educación, 6, 33-42.

Sarrazin, P. y Guillet, E. (2001). "Mais pourquoi ne se réinscrivent-ils plus!" Variables et processus de l'abandon sportif”. En F. Cury, P. y Sarrazin (Eds.), Théories de la motivacion et practiques sportives: état des recherches, 223-254.

Sarrazin, P., Vallerand, R., Guillet, E., Pelletier, L. y Cury, F. (2002). Motivation and dropout in female handballers: A 21-month prospective study. European Journal of Social Psychology, 32(3), 395-418.

Varo, J., Martínez, M., de Irala, J., Kearney, J., Gibney, M. y Martínez, J. (2003). Distribution and determinants of sedentary lifestyles in the European Union. International Journal of Epidemiology, 32(1), 138-146.

Mendoza R., Sagrera, M. y Batista, J. (1994). Conductas de los escolares españoles relacionadas con la salud (1986-1990). Madrid: Consejo Superior de Investigaciones Científicas.

Ministerio de Sanidad y Consumo (2009). Guía para una escuela activa y saludable: Orientación para los Centros de Educación Primaria. Recuperado el 16 de octubre de 2010, de http://www.perseo.aesan.msps.es/docs/ docs/guias/escuela_activa.pdf.

Moreno, J., Gómez, A. y Pérez, A. (2007). Propuesta didáctica sobre la teoría de la necesidad en Educación Física. INDEref-Revista de Educación Física. http://www.inderef.com Potenciado por Joomla! Generado: 16 December, 23:41.

OMS (2004). Estrategia mundial sobre régimen alimentario, actividad fisica y salud. Recuperado el 15 de abril de 2010, de http://www.who.int/dietphysicalactivity/strategy/eb11344/strategy_spanish_web.pdf.

Palou, P., Ponseti, X., Gili, M, Borras, P. y Vidal, J. (2005). Motivos para el inicio, mantenimiento y abandono de la práctica deportiva de los preadolescentes de la isla de Mallorca. Apunts: Educación Física y Deportes, 81, 5-11.

Pérez Samaniego, V.M (1999). El cambio de las actitudes hacia la actividad fisica relacionada con la salud: una investigación con estudiantes de magisterios especialistas en Educación Física. Tesis Doctoral. Universidad de Valencia.

Piéron, M., Telama, R., Almond, L. y Carreiro da Costa, F. (1999). Estilo de vida de jóvenes europeos: Un estudio comparativo. Revista de Educación Física, 76, 5-13.

Quevedo-Blasco, V., Quevedo-Blasco, R. y Bermúdez, M. (2009). Análisis de la motivación en la práctica de actividad físico-deportiva en adolescentes. Revista de Investigación en Educación, 6, 33-42.

Ruiz Juan, F., García Montes, M. y Gómez López, M. (2005). Hábitos físico deportivos en centros escolares y universitarios. Madrid: Gymnos.

Sánchez Bañuelos, F. (1996). La actividad física orientada hacia la salud. Madrid: Biblioteca nueva.

Tercedor, P., González Gross, M., Delgado Fernández, M., Chillón, P., Pérez López, I. J., Ruiz, J. R., et al. (2003). Motives and frequency of physical activity in spanish adolescents. The AVENA study. Annual 
nutrition metabolims, 47, 499.

Torre, E. (1998). La actividad físico-deportiva extraescolar y su interrelación con el área de educación física en el alumnado de enseñanzas medias. Tesis doctoral. Universidad de Granada.
Vicente-Rodríguez, G., Rey-López, J., Martín-Matillas, M., Moreno, L., Wärnberg, J., Redondo, C. (2008). Television watching, videogames, and excess of body fat in Spanish adolescents: the AVENA study. $\mathrm{Nu}$ trition, 24(7-8), 654-662. 